\title{
Characterization of Natural Isolates of Bipolaris maydis Associated with Mating Types, Genetic Diversity, and Pathogenicity in Fujian Province, China
}

\author{
Yuli Dai, Lin Gan, Hongchun Ruan, Niuniu Shi, Yixin Du, Furu Chen, and Xiujuan Yang ${ }^{\dagger}$ \\ Fujian Key Laboratory for Monitoring and Integrated Management of Crop Pests, Institute of Plant Protection, Fujian Academy of \\ Agricultural Sciences, Fuzhou, Fujian Province 350013, China
}

\begin{abstract}
Due to the natural destructiveness and persistence of the southern corn leaf blight (SCLB) fungus Bipolaris maydis (Nisikado et Miyake) Shoem, the characterization of $B$. maydis field isolates is essential to guide the rational distribution of resistant materials in corn-growing regions. In the present study, 102 field isolates collected from seven locations covering the entire region of Fujian Province, China, were assessed for mating type distribution, genetic diversity, and pathogenicity toward local sweet corn cultivars. Mating type detection via polymerase chain reaction indicated that 36.3 and $63.7 \%$ of isolates were MAT1-1 and MAT1-2, respectively; more than $80 \%$ of these isolates were confirmed using cross assays with known mating type isolates. Thirteen intersimple

population was more diverse based on DNA polymorphism than the MAT1-1 population. The value of $G_{S T}$ was 0.0070 , ranging from 0.0399 to 0.3044 based on analysis of combined isolates and individual regional populations, respectively, suggesting the presence of genetic differentiation in the two mating type populations from different locations. Pathogenicity assays revealed that both MAT1-1 and MAT1-2 populations were pathogenic to all 11 local sweet corn cultivars tested in this study. The potential of sexual reproduction, existence of genetic diversity in the two mating type populations, and pathogenicity suggest that $B$. maydis populations have independently clonally adapted under natural field conditions during corn cultivation.
\end{abstract} sequence repeat (ISSR) markers within and among two mating type populations revealed a high level of DNA polymorphism for all combined isolates and between MAT1-1 and MAT1-2 populations. The MAT1-2
Keywords: Bipolaris maydis, mating type, genetic diversity, pathogenicity, southern corn leaf blight
Southern corn leaf blight (SCLB), caused by the filamentous fungus Bipolaris maydis (Nisikado et Miyake) Shoem (teleomorph: Cochliobolus heterostrophus [Drechsler] Drechsler), is a primary and challenging disease that significantly affects corn (Zea mays L.) production worldwide. Corn is a crucial cereal crop and an important source of industrial raw material and livestock fodder (Zwonitzer et al. 2009). However, SCLB is a major limitation to yields in cornproducing regions, with serious losses of $10 \%$ to $68 \%$ in epidemic years (Dai et al. 2016; Ngoko et al. 2002). Airborne conidia produced on the lower leaves by the initial infection spread through airflow and rain splashes, causing secondary infections under temperate and moist conditions (Aylor and Lukens 1974; Carson 1998; Dai et al. 2018a). Unfortunately, these meteorological conditions are particularly pronounced in Fujian Province, where SCLB is a new disease and has become a major constraint to corn production (Dai et al. 2016). Furthermore, an increasing challenge to SCLB management in Fujian Province is the occurrence of resistance to demethylation inhibitor fungicides among B. maydis populations (Gan et al. 2016).

${ }^{\dagger}$ Corresponding author: X. Yang; yxjzb@126.com

Funding: This study is supported by the Natural Science Foundation of Fujian Province, China (grant no. 2016J05073), the Special Fund for Agro-scientific Research in the Public Interest of Fujian Province, China (2017R1025-3 and 2018R1025-1), the National Key Research and Development Program of China (2018YFD0200706), the 100 Youth Science and Technology Talents Project of Fujian Academy of Agricultural Sciences (YC2016-4), the Youth Freedom Exploration Project of Fujian Academy of Agricultural Sciences (AA2018-14), and Innovation Team of Plant Protection, Fujian Academy of Agricultural Sciences (STIT2017-1-8).

The author(s) declare no conflict of interest.

Accepted for publication 15 September 2019.

(c) 2020 The American Phytopathological Society
The underlying mechanisms of sexual reproduction among $B$. maydis populations in the context of SCLB management and epidemiology need to be thoroughly elucidated. Sexual recombination plays key roles in producing genetic diversity and generating new genotypes that cause plant-pathogenic fungi to overcome host resistance or become resistant to fungicides (Bi et al. 2014; Glass and Kuldau 1992). Nevertheless, the sexual phase for most ascomycetous phytopathogens, including B. maydis, has rarely been observed under natural field or even laboratory conditions (Kim et al. 2013). Similar to other heterothallic plant pathogens, sexual reproduction in B. maydis is dependent on coexistence and mating between individuals with opposite idiomorphs at the single mating type (MAT) locus, termed MAT1-1 and MAT1-2 (Turgeon et al. 1993). Amphiprotic isolates of B. maydis have been identified in the United States (Leonard 1974), Japan (Tsukiboshi et al. 1996), and China (Cui and Huang 1985) and used as standard isolates for differentiating the mating types of $B$. maydis. Indeed, the mating type genes of $B$. maydis have been characterized and cloned (Turgeon et al. 1993; Wirsel et al. 1998), and a molecular approach for detecting B. maydis mating types was established 20 years ago (Gafur et al. 1997). Regardless, there are only limited studies to date on the distribution of $B$. maydis mating types in epidemic populations under natural field conditions.

Because the perfect stage of $B$. maydis rarely occurs in nature and is difficult to obtain in laboratory crosses, investigations of matingtype frequencies, genetic diversity, and population differentiation are beneficial for researchers to reveal the existence of sexual recombination (Carson 1998; Milgroom 1996). In general, higher genetic diversity and equal frequencies between the two mating types are observed in sexually reproducing populations when compared with asexual reproducing populations (Groenewald et al. 2008; Grünwald et al. 2003; Milgroom 1996). However, current studies concerning $B$. maydis focused on its pathogenesis and control, and there are few studies on the diversity of B. maydis. Molecular markers have been broadly applied to the identification, species evolution, and genetic diversity of the plant pathogen (Ahmadpour et al. 2018; Reddy 
et al. 2012; Sharma et al. 2018). Genetic variation in several populations of $B$. maydis from different countries has been successfully studied using several molecular markers, including random amplified polymorphic DNA (RAPD) (Karimishahri and Sharma 2016; Nicholson et al. 1993), amplified fragment length polymorphism (Wang et al. 2017), and restriction fragment length polymorphism (RFLP) (Tzeng et al. 1992). Owing to the simultaneous analysis of abundant banding profiles and high repeatability compared with RAPD or RFLP, intersimple sequence repeat (ISSR) molecular markers provide noteworthy sensitivity and reliability and are suitable for use in genetic diversity analysis (Kantety et al. 1995; Salimath et al. 1995). Because of these advantages, the ISSR technique has been proposed as a useful tool for evaluating genetic diversity and population structure (Li et al. 2012). However, this powerful molecular technique has not been implemented for evaluating the genetic diversity between $B$. maydis mating type populations.

Many inbred lines with cms-T (Texas male sterile cytoplasm), cms-C, cms-S, and normal (N) cytoplasm have been employed in the United States and China to characterize pathotypes of B. maydis populations when selecting resistant corn materials (Kong et al. 2005; Smith et al. 1970; Wang et al. 2017). In China, it was recently demonstrated that the proportion and distribution of a specific pathotype (race $\mathrm{O}$ ) of $B$. maydis rapidly increases after corn varieties with cms-T are replaced with those with normal cytoplasm (Wang et al. 2010, 2017; Zhao et al. 2012). Although sweet corn hybrids with high quality and yield are planted throughout Fujian Province, there is a lack of systematic studies on the adaptation of the two mating type populations of $B$. maydis to these sweet corn varieties.

Therefore, the main objectives of the present study were to (i) determine the distribution of mating types of $B$. maydis isolates covering seven sweet corn-growing regions in Fujian Province, (ii) determine the genetic diversity of MAT1-1 and MAT1-2 populations using ISSR markers, and (iii) assess the pathogenicity and fitness of these mating type populations in regard to 11 local sweet corn cultivars. The results of the present study will enhance the current understanding of the potential role of sexual recombination and our understanding of diversity within the population in the causal fungus of SCLB in Fujian Province.

\section{Materials and Methods}

Isolation of $\boldsymbol{B}$. maydis isolates. Bipolaris maydis isolates were collected from seven sweet corn-growing regions in Nanping (NP), Ningde (ND), Fuzhou (FZ), Putian (PT), Sanming (SM), Longyan (LY), and Zhangzhou (ZZ) in Fujian Province, China. One corn leaf with typical symptoms of SCLB was randomly collected from each field; the plant was either a seedling or approaching maturity, and at least nine fields were sampled in each region. Five isolates were obtained from different lesions on each leaf by surface sterilizing lesions $(5 \times 5 \mathrm{~mm})$ in $75 \%(\mathrm{v} / \mathrm{v})$ alcohol for $2 \mathrm{~min}$ and in $0.1 \%(\mathrm{w} / \mathrm{v})$ mercuric chloride solution for $90 \mathrm{~s}$, followed by rinsing three times in distilled water and air drying on sterile gauze. The samples were placed on potato dextrose agar (PDA; potato, $200 \mathrm{~g} /$ liter; dextrose, $20 \mathrm{~g} /$ liter; agar, $18 \mathrm{~g} /$ liter) and incubated at $28^{\circ} \mathrm{C}$ in darkness for 3 days (Dai et al. 2016). An agar plug (6 $\mathrm{mm}$ in diameter) containing active growing mycelia from the margin of 3-day-old colonies of each isolate was inoculated at the center of the fresh PDA plate. The plates were incubated in the dark at $28^{\circ} \mathrm{C}$ for 7 days. Conidia of each isolate were washed with $5 \mathrm{ml}$ of sterilized water from the PDA plate and filtered through double-layer sterile gauze (Xuzhou Weicai Hygienic Material, Xuzhou, China). The concentration of conidia in each isolate was adjusted to $1.0 \times 10^{5}$ conidia $/ \mathrm{ml}$ using a hemocytometer (Refined Biochemical Reagent Instrument Co., Shanghai, China) under an Olympus microscope $(10 \times 20$ magnification; Olympus Corporation, Tokyo, Japan). One hundred microliters of conidial suspension $\left(1.0 \times 10^{5}\right.$ conidia/ml $)$ of each isolate was spread on water agar (WA; water, 1,000 ml; agar, $18 \mathrm{~g}$ ) and incubated in the dark at $28^{\circ} \mathrm{C}$ for 12 to $24 \mathrm{~h}$. Three WA plates were done for each isolate. A small piece of agar containing a germinated spore was transferred into a fresh PDA plate (five spores per plate), and the inoculated plates were incubated in the dark at $28^{\circ} \mathrm{C}$ for 5 days
(Gao et al. 2010). Finally, one pure monosporic isolate from each leaf that was sampled was arbitrarily selected, and a total of 102 natural isolates were obtained from NP (15 isolates), ND (16 isolates), FZ (10 isolates), PT (15 isolates), SM (13 isolates), LY (9 isolates), and ZZ (24 isolates) in Fujian Province, China. These monosporic isolates were used for mating type distribution, genetic diversity, and pathogenicity assays in the present study. Mating type distribution within field was additionally studied using 96 isolates collected from three sweet corn fields growing the same corn variety, Shangpin, in Fuqing (34 isolates), Jian'ou (JO; 32 isolates), and Pingnan (PN; 30 isolates) prefectures of Fujian Province. Thirty to 34 SCLB-symptomatic corn leaves were randomly collected at each field when corn approached maturity. One isolate was recovered from each leaf as described above. Singlespore isolates were cultured on filter papers on PDA and enclosed in small sterilized sealed envelopes for long-term storage at $-20^{\circ} \mathrm{C}$.

Cross assays. Two tester isolates, PN1606 (MAT1-1) and JO1601DT09 (MAT1-2), obtained from naturally infected corn leaves in Fujian Province, were used for detecting mating types. The two isolates can mate and easily produce perithecia. Crosses were performed as previously described by Wirsel et al. (1996), with a few modifications. In general, a sterilized senescent corn leaf piece $(30 \times 50 \mathrm{~mm})$ was placed at the center of the 9-cm Petri dishes containing $20 \mathrm{ml}$ of modified Sach's agar (potato, $200 \mathrm{~g} / \mathrm{liter} ; \mathrm{K}_{2} \mathrm{HPO}_{3}$, 0.25 g/liter; $\mathrm{CaCO}_{3}, 4.00$ g/liter; $\mathrm{Ca}\left(\mathrm{NO}_{3}\right)_{2}, 1.00$ g/liter; $\mathrm{MgSO}_{4}$, $0.25 \mathrm{~g} / \mathrm{liter} ; \mathrm{FeCl}_{3}, 0.005 \mathrm{~g} / \mathrm{liter}$; and agar, $18 \mathrm{~g} / \mathrm{liter}$ ). Two plugs (6 $\mathrm{mm}$ in diameter) containing fresh mycelia from the margin of 3day-old colonies of tester isolate (MAT1-1 or MAT1-2) were inoculated on the surface of the Sach's medium at one side of the sterilized leaf piece. Two plugs containing fresh mycelia from a mating typeunknown $B$. maydis isolate were placed symmetrically on opposite sides of the senescent corn leaf piece. Each isolate contained three repetitions. The inoculated plates were incubated at $24^{\circ} \mathrm{C}$ in darkness. Mature perithecia at the intersection of growth on the leaves were assessed after 20 to 25 days (Wirsel et al. 1996). Fertility between the unknown mating-type isolate and the two testers was defined as the generation of mature perithecium-producing ascospores. Field isolates unable to mate with the pair of standard isolates were recorded as "mating type unknown."

DNA extraction and mating type polymerase chain reaction (PCR). The 198 B. maydis isolates that were evaluated were each grown on PDA at $28^{\circ} \mathrm{C}$ for 7 days in the dark. Each isolate was inoculated onto three plates. Mycelia of each isolate was scraped from each plate and transferred into a separate 2-ml tube. One tube of each isolate was randomly selected for DNA extraction, and the other two tubes were stored at $-80^{\circ} \mathrm{C}$ immediately. DNA from each isolate was extracted from mycelia using the modified cetyltrimethylammonium bromide (Shanghai Sangon Biotech, Shanghai, China) method (Neuhauser et al. 2009). Two pairs of mating type-specific primers (ChMAT01-1 for MAT1-1 and ChMAT02-1 for MAT1-2) were used to determine mating types of B. maydis (Dai et al. 2018a). Mating type-specific sequences were amplified by PCR using the following primers: ChMAT01-1 (ChMAT01-1F, 5'-AGAAACCAC GAGACAACAT-3'; ChMAT01-1R, 5'-CGTAACATAGTAGCC TAAACAC-3'), with a PCR product length of 307 bp for MAT1-1, and ChMAT02-1 (ChMAT02-1F, 5'-AAGCCTGGCGAGAAGAAA3'; ChMAT02-1R, 5'-GGCGGATTGACTCAGTGTTG-3'), with a product length of 283 bp for MAT1-2. PCR was performed using $2 \times$ Premix Taq MasterMix (Takara Biotech, Dalian, China); each 25- $\mu 1$ PCR reaction contained $12.5 \mu \mathrm{l}$ of Premix Taq, 10 pmol of primers, and $20 \mathrm{ng}$ of DNA templates. Amplifications were performed using a Thermal Cycler C1000 PCR System (Bio-Rad Laboratories, Hercules, CA) with initial denaturation at $95^{\circ} \mathrm{C}$ for $5 \mathrm{~min}$, followed by 35 cycles at $95^{\circ} \mathrm{C}$ for $45 \mathrm{~s}, 55^{\circ} \mathrm{C}$ for $45 \mathrm{~s}$, and $72^{\circ} \mathrm{C}$ for $45 \mathrm{~s}$, and a final cycle at $72^{\circ} \mathrm{C}$ for $10 \mathrm{~min}$. The amplification products were analyzed by $1.0 \%$ agarose gel electrophoresis (Biowest, Nuaillé, France) and stained with ethidium bromide (Shanghai Sangon Biotech). PCR for each isolate was performed three times. The fragment sizes of the products were estimated using a 2-kb DNA ladder (Takara Biotech).

Genetic diversity analysis using ISSR markers. A total of 13 highly polymorphic ISSR primers, initially screened from the ISSR 
primer database developed by the University of British Columbia (UBC) Biotechnology Laboratory (UBC Primer Set 9; Vancouver, $\mathrm{BC}$, Canada), were selected for further diversity analysis (Table 1). Primers DC02, DC03, and DC05 were optimized from primers UBC841, UBC856, and UBC848, respectively, by modifying one side of the primer. The annealing temperature and ISSR-PCR reaction system for each primer were optimized using 15 DNA samples prior to using the ISSR markers for all samples. The ISSR-PCR reaction involved a $25-\mu l$ amplification system comprising $12.5 \mu \mathrm{l}$ of Premix Taq MasterMix (Takara Biotech), 10 pmol of primers, and $100 \mathrm{ng}$ of DNA template. ISSR-PCR amplifications were performed using a Thermal Cycler C1000 PCR System (Bio-Rad Laboratories) with initial denaturation at $94^{\circ} \mathrm{C}$ for $5 \mathrm{~min}$, followed by 35 cycles at $94^{\circ} \mathrm{C}$ for $45 \mathrm{~s}$, a specific annealing temperature for each primer for $60 \mathrm{~s}$, and $72^{\circ} \mathrm{C}$ for $90 \mathrm{~s}$, and a final cycle at $72^{\circ} \mathrm{C}$ for $10 \mathrm{~min}$. The products were separated with $2.0 \%$ agarose gel electrophoresis containing ethidium bromide (Shanghai Sangon Biotech), visualized on a Benchtop 3UV transilluminator (UVP, Upland, CA), and imaged using a BioDoc-It Imaging System (UVP). Fragment sizes for each ISSR locus across the samples were recorded and used for statistical analysis.

Corn cultivars and pathogenicity assays. Two resistant sweet corn cultivars, Rongtian 1 (Fujian Nongfeng Agricultural Development) and Yongzhen 7 (Fujian Chaoda Modern Seed Industry), and nine moderately susceptible or susceptible sweet corn cultivars, Mintian 4 (Institute of Crop Research, Fujian Academy of Agricultural Sciences), Yuetian 16 (Institute of Crop Research, Guangdong Academy of Agricultural Sciences), Shangpin (Fujian Nongfeng Agricultural Development), Xianyunuo 4 (Institute of Cereal Research, Hainan Academy of Agricultural Sciences), Meiyu 8 (Hainan Lvchuan Seeds), Suyunuo 5 (Jiangsu Yanjiang Institute of Agricultural Sciences), Hongyu 2 (Nanning Guifuyuan Agricultural Co.), Jingkenuo 2000 (Maize Research Center, Beijing Academy of Agriculture and Forestry Sciences), and Nongkeyu 368 (Maize Research Center), were provided by Fujian Seed Management Station. The tested sweet corn cultivars are popular and grown throughout Fujian Province. One hundred two single-spore isolates were cultured at $28^{\circ} \mathrm{C}$ under dark conditions in 9-cm Petri plates containing PDA medium for conidial production and plant inoculation. Pathogenicity assays were performed as previously described by Dai et al. (2019). Briefly, corn seeds were sown in seedling-raising plates and grown to the three- to four-leaf stage, and the seedlings were then transplanted into plastic flowerpots (one plant per pot) and grown to the seven- to eight-leaf stage. Subsequently, the corn plants were inoculated by spraying conidial suspension $\left(5 \times 10^{5}\right.$ conidia/ml $)$ until runoff. The inoculated plants were maintained at $28 / 25^{\circ} \mathrm{C}$ (day/night) in a greenhouse (12 h light; relative humidity $>90 \%$ ) for 10 days. Each $B$. maydis isolate was inoculated onto 20 plants of each sweet corn cultivar, and there were three replicates of each B. maydis-sweet corn cultivar combination for a total of 60 plants. The experiment was independently performed twice. Disease severity was evaluated at 10 days post-inoculation using a 0 to 9 scale $(0=$ no evidence of the leaf surface diseased; $1=$ no more than $5 \%$ of the leaf surface diseased; $3=6$ to $10 \%$ of the leaf surface diseased; $5=11$ to $30 \%$ of the leaf surface diseased; $7=31$ to $70 \%$ of the leaf surface diseased; $9=$ over $70 \%$ of the leaf surface diseased), and disease index (DI) for each isolate on each cultivar was calculated using the following formula:

$$
\text { DI }(\%)=\frac{\sum\left(n_{i} \times i\right)}{N \times 9} \times 100
$$

where DI is disease index, $n_{i}$ is the number of diseased plants in the severity grade of $i, i$ is disease severity in the grade of $i$, and $N$ is the total investigated plants.

An isolate of B. maydis was defined as pathogenic on a cultivar when disease severity was $\geq 5$ and disease index was $>35$ (Dai et al. 2019). The frequencies of pathogenic isolates on each cultivar were calculated.

Data analysis. The ISSR-PCR amplification results across the samples for each primer were recorded as 1 (presence) or 0 (absence) according to DNA fingerprints. ISSR marker genetic diversity was analyzed using POPGENE version 1.32 software, as previously described ( $\mathrm{Li}$ et al. 2016; Nei 1973; Yeh et al. 1999). The number of loci $(\mathrm{N})$, number of polymorphic loci (NPL), percentage of polymorphic loci (PPL), Nei's gene diversity (h), Shannon's information index (I), observed number of alleles (na), effective number of alleles (ne), total genetic diversity, genetic differentiation $\left(G_{S T}\right)$, genetic similarity, and genetic distance were automatically generated using POPGENE version 1.32 software on the basis of bootstrap analysis of the binary matrix ISSR data with 1,000 replicates (Yeh et al. 1999). To calculate $G_{S T}$ for ISSR loci between the opposite mating type groups, total genetic diversity was divided into within and among mating types (McDermott and McDonald 1993). Genetic differentiation among the two mating type groups was estimated based on the value of $G_{S T}\left(G_{S T}<\right.$ 0.05 is low, 0.05 to 0.15 is medium, and $>0.15$ is high) ( $\mathrm{Li}$ et al. 2016; Onaga et al. 2015). Genetic similarity coefficients were used for constructing a phylogram using the genetic arithmetic of unweighted pair-group method with arithmetic averages with NTSYS-pc version 2.10e (Li et al. 2012).

Because no significant differences were detected among pathogenicity experiments $(P<0.01)$, disease severity data from each experiment were averaged for analysis of variance. The least square means of the mean disease severity value from each isolate on each corn cultivar were calculated using the PROC GLM procedure in DPS (version 7.05, RuiFeng Information Technology, Hangzhou, China) with isolate, MAT, cultivar, isolate $\times$ cultivar, and MAT $\times$ cultivar considered as random effects. Differences in the random-effect variation were analyzed using the method of least significant difference $(P<0.01$; PROC GLM, type III) (Balint-Kurti et al. 2008; Tang 2010).

Table 1. Primers used for intersimple sequence repeat markers in the present study, showing primer codes, sequences, annealing temperatures, number of loci ( $\mathrm{N}$ ), and number of polymorphic loci (NPL) $)^{\mathrm{a}}$

\begin{tabular}{llrr}
\hline Primer codes & Primer sequences $\left(\mathbf{5}^{\prime} \rightarrow \mathbf{3}^{\prime}\right)$ & Annealing temperature & N \\
\hline UBC808 & AGAGAGAGAGAGAGAGC & $52^{\circ} \mathrm{C}$ & 12 \\
UBC835 & AGAGAGAGAGAGAGAGYC & $55^{\circ} \mathrm{C}$ & 12 \\
UBC841 & GAGAGAGAGAGAGAGAYC & $52^{\circ} \mathrm{C}$ & 22 \\
UBC856 & ACACACACACACACACYA & $50^{\circ} \mathrm{C}$ & 15 \\
UBC857 & ACACACACACACACACYG & $53^{\circ} \mathrm{C}$ & 13 \\
UBC859 & TGTGTGTGTGTGTGTGRC & $51^{\circ} \mathrm{C}$ & 18 \\
UBC884 & HBHAGAGAGAGAGAGAG & $53^{\circ} \mathrm{C}$ & 19 \\
UBC888 & BDBCACACACACACACA & $53^{\circ} \mathrm{C}$ & 18 \\
UBC889 & DBDACACACACACACAC & $51^{\circ} \mathrm{C}$ & 19 \\
UBC891 & HVHTGTGTGTGTGTGTG & $50^{\circ} \mathrm{C}$ & 17 \\
DC02 & GAGAGAGAGAGAGAGAHC & 17 \\
DC03 & ACACACACACACACACYC & 16 \\
DC05 & BVBCACACACACACACA & $53^{\circ} \mathrm{C}$ & 11 \\
Total & $\ldots$ & $51^{\circ} \mathrm{C}$ & 18 \\
\hline
\end{tabular}

${ }^{a} \mathrm{Y}=\mathrm{C}+\mathrm{T} ; \mathrm{R}=\mathrm{A}+\mathrm{G} ; \mathrm{H}=\mathrm{A}+\mathrm{T}+\mathrm{C} ; \mathrm{B}=\mathrm{T}+\mathrm{C}+\mathrm{G} ; \mathrm{D}=\mathrm{A}+\mathrm{T}+\mathrm{G}$; and $\mathrm{V}=\mathrm{A}+\mathrm{C}+\mathrm{G}$. Primers $\mathrm{DC} 02$, DC03, and DC05 were optimized from primers $\mathrm{UBC} 841$, UBC856, and UBC848 (CACACACACACACACARG), respectively, by modifying one side of the primer. 


\section{Results}

Mating type distribution in Fujian Province. In the present study, mating type was successfully identified in more than $80 \%$ of B. maydis isolates by using the two tester isolates (data not shown). The mating types for all tested isolates were reconfirmed by PCR assays with mating type-specific primers. Overall, 37 (36.3\%) isolates were verified as MAT1-1 and $65(63.7 \%)$ isolates as MAT1-2 in Fujian Province (Fig. 1). The two mating types of B. maydis were also identified throughout the province (Fig. 1), and more than $60 \%$ of isolates in FZ, PT, ZZ, LY, SM, and ND were characterized as MAT1-2. For NP, the abundance of isolates of the two mating types was nearly 1:1 (Fig. 1). The distribution of MAT1-1 and MAT1-2 was unsymmetrical in specific field conditions (Table 2). The results demonstrated that the two mating types of $B$. maydis coexist in Fujian Province, even in small field conditions (Table 2; Fig. 1 ). To date, no sexual isolates of $B$. maydis have been detected under natural conditions, suggesting that sexual reproduction did not occur in fields in present study.

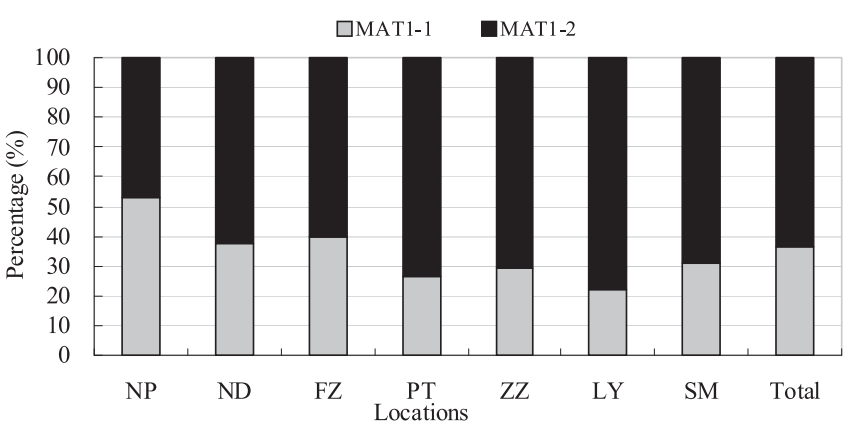

Fig. 1. Distribution of mating types (MAT1-1 and MAT1-2) of Bipolaris maydis in Fujian Province of China. NP, ND, FZ, PT, ZZ, LY, and SM represent $B$. maydis isolates collected from the Nanping (15 isolates), Ningde (16 isolates), Fuzhou (10 isolates), Putian (15 isolates), Zhangzhou (24 isolates), Longyan (9 isolates), and Sanming (13 isolates) regions of Fujian Province, respectively. The mating type of 102 isolates was detected using a PCR-based method.

Table 2. Mating types of Bipolaris maydis recovered from three fields detected using mating type-specific primers in the polymerase chain reaction

\begin{tabular}{lccc}
\hline & \multicolumn{2}{c}{$\begin{array}{c}\text { Number and proportion of } \\
\text { MAT1-1 and MAT1-2 isolates }\end{array}$} & \\
\cline { 2 - 3 } Fields & MAT1-1 & MAT1-2 & Total isolates \\
\hline Pingnan & $11(36.7 \%)$ & $19(63.3 \%)$ & 30 \\
Fuqing & $15(44.1 \%)$ & $19(55.9 \%)$ & 34 \\
Jian'ou & $16(50.0 \%)$ & $16(50.0 \%)$ & 32 \\
Total & $42(43.8 \%)$ & $54(56.2 \%)$ & 96 \\
\hline
\end{tabular}

Genetic diversity and differentiation between MAT1-1 and MAT1-2 populations. To determine genetic diversity among populations of $B$. maydis mating types, 13 ISSR-PCR primers were used to amplify 37 isolates for MAT1-1 and 65 isolates for MAT1-2, covering seven locations in Fujian Province. The values of N, NPL, PPL, na, ne, h, and I were calculated (Table 3). The 13 ISSR-PCR primers amplified a total of 198 repeatable loci from the MAT1-1 and MAT1-2 populations, of which 178 loci for the MAT1-1 population and 189 loci for the MAT1-2 population were polymorphic (89.9 and $95.5 \%$, respectively) (Table 3). The values of NPL and PPL for the MAT1-2 population were higher than those for the MAT1-1 population (Table 3), suggesting that the MAT12 population was more diverse on DNA polymorphism than was the MAT1-1 population.

$G_{S T}$ for ISSR markers between the MAT1-1 and MAT1-2 populations ranged from 0.0399 to 0.3044 according to an analysis of individual regional populations and was 0.0700 when the analysis was based on combined isolates (Table 4). Phylogenetic analysis showed that the MAT1-1 and MAT1-2 populations from the same location were randomly distributed into different clusters, with different locations grouping on the same branch (Fig. 2), suggesting the certain existence of genetic differentiation in the two mating type populations from different locations.

Pathogenicity of the two mating type populations. Both MAT1-1 and MAT1-2 populations were pathogenic on 11 tested sweet corn cultivars. In the both MAT1-1 and MAT1-2 populations, disease severity $\geq 5$ (disease index $>35$ ) on the two resistant cultivars for more than $64 \%$ of isolates, and disease severity $\geq 7$ (disease index $>35$ ) on the nine susceptible cultivars for more than $87 \%$ of isolates (Fig. 3). Analysis of variance, using isolate as an independent variable (Table 5), showed highly significant differences among isolates $(P<0.001)$. Despite the consistent differences in disease severity (Table 5), no difference in aggressiveness among the two mating type populations was detected $(P>0.625)$. Isolate $\times$ cultivar $(P>0.997)$ and MAT $\times$ cultivar $(P>0.637)$ interactions were nonsignificant at the level of $99 \%$ (Table 5), suggesting that MAT1-1 and MAT1-2 populations exhibit analogous pathogenicity. Notably, frequencies of pathogenic isolates on resistant cultivars were considerably lower than those on susceptible cultivars, regardless of mating type category (Fig. 3). A significant difference $(P<0.001)$ was detected among cultivars (Table 5).

\section{Discussion}

Both MAT1-1 and MAT1-2 were detected in seven regions at a large-scale geographical distribution, including the coexistence of MAT1-1 and MAT1-2 within three specific fields, suggesting that coexistence of opposite mating types is widespread in natural field conditions. Intriguingly, MAT1-1 and MAT1-2 exhibited obvious location-specific differences and a skewed proportion toward

Table 3. Genetic diversity between MAT1-1 and MAT1-2 populations in Fujian Province based on intersimple sequence repeat (ISSR) markers ${ }^{\mathrm{a}}$

\begin{tabular}{lcccccccc}
\hline MAT & $\boldsymbol{n}$ & $\mathbf{N}$ & NPL & PPL & na & ne & h \\
\hline MAT1-1 & 37 & 198 & 178 & $89.9 \%$ & $1.90 \pm 0.30$ & $1.35 \pm 0.32$ & $0.22 \pm 0.17$ & $0.35 \pm 0.23$ \\
MAT1-2 & 65 & 198 & 189 & $95.5 \%$ & $1.95 \pm 0.21$ & $1.35 \pm 0.33$ & $0.22 \pm 0.17$ & $0.35 \pm 0.23$ \\
Total & 102 & 198 & 193 & $97.5 \%$ & $1.97 \pm 0.16$ & $1.36 \pm 0.33$ & $0.22 \pm 0.17$ & $0.36 \pm 0.23$ \\
\hline
\end{tabular}

a MAT = mating type; $n=$ number of isolates; $\mathrm{N}=$ number of loci; NPL = number of polymorphic loci; PPL = percentage of polymorphic loci; na $=$ observed number of alleles; ne = effective number of alleles; $\mathrm{h}=$ Nei's gene diversity (Nei 1973); and I = Shannon's information index (Lewontin 1972). Numbers were calculated by bootstrap analysis of the original ISSR data with 1,000 replicates using POPGENE version 1.32 software (Yeh et al. 1999).

Table 4. Genetic differentiation $\left(G_{S T}\right)$ between MAT1-1 and MAT1-2 populations in Fujian Province using intersimple sequence repeat markers ${ }^{\mathrm{a}}$

\begin{tabular}{lcccccrrr}
\hline & \multicolumn{7}{c}{ Genetic differentiation for seven locations in Fujian Province } \\
\cline { 2 - 9 } Parameter & FZ & PT & ZZ & LY & SM & NP & ND \\
\hline$G_{S T}$ & 0.3044 & 0.0859 & 0.0500 & 0.2100 & 0.1330 & 0.0568 & 0.0399 \\
Nm & 1.1428 & 5.3219 & 9.4921 & 1.8815 & 3.2600 & 8.2971 & 12.0462 \\
\hline
\end{tabular}

${ }^{a}$ FZ, PT, ZZ, LY, SM, NP, and ND represent Bipolaris maydis isolates collected from the Fuzhou, Putian, Zhangzhou, Longyan, Sanming, Nanping, and Ningde regions of Fujian Province, respectively. $\mathrm{Nm}=$ estimate of gene flow from $G_{S T} . \mathrm{Nm}=0.5\left(1-G_{S T}\right) / G_{S T}$ (McDermott and McDonald 1993). 
MAT1-2 in most Fujian populations (ND, FZ, PT, ZZ, LY, and SM; Fig. 1), which suggests that the MAT1-2 population may be more adaptive in Fujian Province, although the regions from which isolates of $B$. maydis were collected are separated by mountain ranges. The ability of $B$. maydis to complete sexual reproduction will depend on factors besides the presence of the two opposite mating types

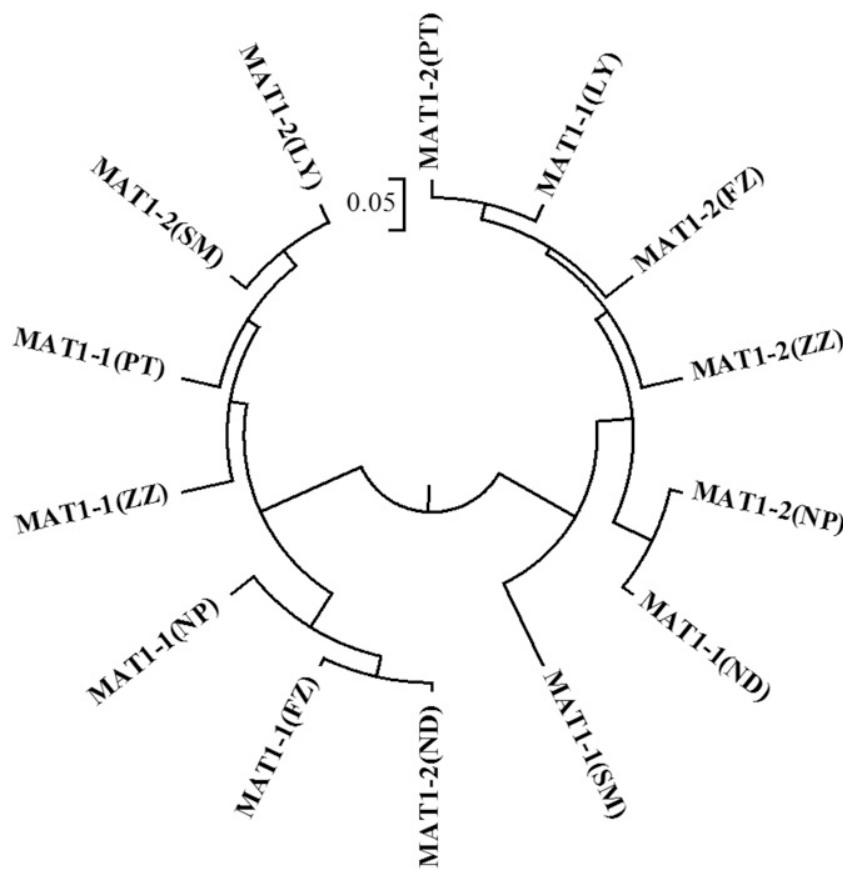

Fig. 2. Phylogram of the two mating type populations from different locations based on intersimple sequence repeat markers. FZ, PT, ZZ, LY, SM, NP, and ND indicate the Fuzhou, Putian, Zhangzhou, Longyan, Sanming, Nanping, and Ningde regions of Fujian Province, respectively. MAT1-1 and MAT1-2 isolates were randomly distributed into different clusters, suggesting that the genetic variation exists between the opposite mating type groups from different locations in Fujian Province.

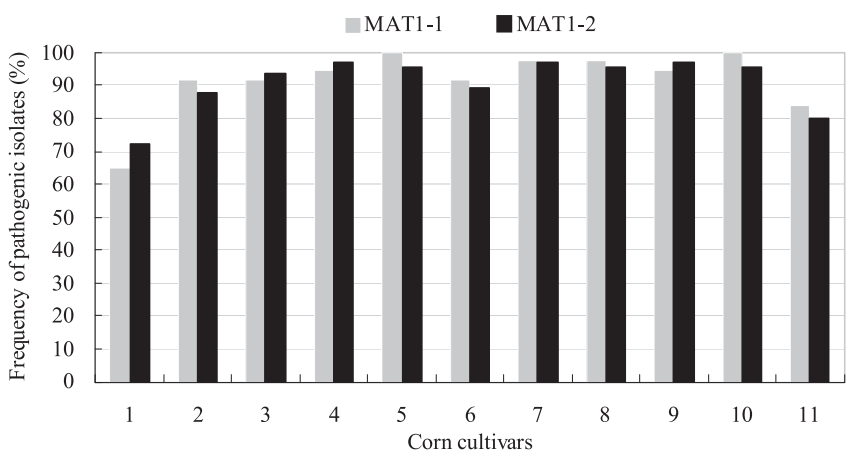

Fig. 3. Pathogenicity of mating type (MAT1-1 and MAT1-2) populations to 11 different sweet corn cultivars. The $x$ axis indicates the 11 different sweet corn cultivars: numbers 1 to 11 represent Rongtian 1 (resistant), Mintian 4, Yuetian 16, Shangpin, Xianyunuo 4, Meiyu 8, Suyunuo 5, Hongyu 2, Jingkenuo 2000, Nongkeyu 368, and Yongzhen 7 (resistant), respectively. The $y$ axis indicates the frequency of pathogenic isolates. within the Fujian population, such as fertility among compatible isolates from different regions, the presence of suitable inductive materials and nutritive substances, and proper environmental conditions. The hypothesis supports the data from our cross assays in which more than $80 \%$ of isolates belonging to the two mating types recovered from different regions in Fujian Province underwent sexual reproduction in laboratory conditions.

The presence of the two opposite mating types is not adequate for demonstration of the existence of sexual reproduction (Linde et al. 2003). An equal mating type frequency across large geographical scales usually implies the occurrence of sexual reproduction owing to frequency-dependent selection (May et al. 1999; Milgroom 1996). Unfortunately, unequal mating type frequencies for most regional populations in the present study are inconsistent with frequency-dependent selection (Linde et al. 2003; May et al. 1999). Thus far, no sexual isolates have been detected among the 198 natural isolates of $B$. maydis, suggesting that sexual reproduction did not occur under natural conditions in Fujian Province. There are two main reasons why no sexual isolates were detected in this study. First, the B. maydis isolates investigated in this study were collected from sweet corn fields in which plants were either seedlings or approaching maturity and had several or many lesions per leaf. Second, senescent leaves are not usually present in the corn fields because the corn residue is removed from the field for continuous cropping.

Regarding the mating type distribution of phytopathogens, it is common for one mating type to be predominant in field populations worldwide. Mazáková et al. (2010) demonstrated that the mating type distribution of late blight pathogen, Phytophthora infestans, is asymmetric between $\mathrm{A} 1$ and $\mathrm{A} 2$ in a Czech population, and Ali et al. (2012) showed that the mating type distribution of the chickpea blight fungus Ascochyta rabiei was skewed in a Pakistani population. Gramaje et al. (2013) found unequal spatial-scale distribution of opposite mating types of Togninia minima in Valencia and Zaragoza. In addition, $\mathrm{Li}$ et al. (2016) reported that more than $60 \%$ of Magnaporthe oryzae MAT1-2 isolates were detectable throughout Yunnan Province, China, whereas most MAT1-1 isolates were found in four regions of the province. Such an asymmetric distribution of B. maydis mating type populations has also been reported in the United States (Fisher and Hooker 1975; Nelson et al. 1971) and Australia (Alcorn 1975). In the present study, we found MAT1-2 was predominant in Fujian Province (Fig. 1), consistent with the results reported by Nelson et al. (1971), who found 33 MAT1-1 isolates and 901 MAT1-2 isolates within a population of B. maydis recovered from the northeastern United States. However, our results are inconsistent with those populations from other provinces of China and Japan, where the two mating types of $B$. maydis were uniformly distributed (Cui and Huang 1985; Tsukiboshi et al. 1996). Furthermore, although an unequal proportion of mating types of $B$. maydis occurred among fields (Table 2), the ratio of mating types of $B$. maydis on a single plant or a single leaf needs further investigation, because the co-occurrence of compatible isolates having different mating types within the same microgeographic scale (e.g., within a single lesion or a single leaf) will theoretically enhance a possibility for random mating interaction (Linde et al. 2003).

The obvious diversity in mating type distribution among most Fujian populations suggests that Fujian isolates of $B$. maydis may have derived from other regions; further confirmation likely requires large-scale sampling from regions other than Fujian. Studies in these

Table 5. Analysis of variance of disease severity from inoculations of 102 Bipolaris maydis isolates representing two mating type (MAT) populations on 11 sweet corn cultivars

\begin{tabular}{|c|c|c|c|c|c|}
\hline Source & Sum of squares & Degrees of freedom & Mean square & $F$ value & $P$ value \\
\hline Isolate & $57,615.65$ & 101 & 570.45 & 7.57 & $<0.001$ \\
\hline MAT & 17.99 & 1 & 17.99 & 0.24 & 0.625 \\
\hline Cultivar & $11,567.03$ & 10 & $1,156.70$ & 15.36 & $<0.001$ \\
\hline Isolate $\times$ cultivar & $25,853.65$ & 1,010 & 25.60 & 0.34 & 0.997 \\
\hline MAT $\times$ cultivar & 596.00 & 10 & 59.60 & 0.79 & 0.637 \\
\hline
\end{tabular}


areas will enhance the current understanding of the origin and distribution of B. maydis in Fujian, where SCLB is a relatively new disease (Dai et al. 2016).

Studies on the distribution of mating types of plant fungal pathogens are of significant importance for inferring levels of genetic diversity owing to potential sexual recombination (Valent et al. 1991). A previous similar investigation indicated that the high level of genetic diversity in Exserohilum turcicum populations in northern China was significantly correlated with mating type (Gu et al. 2008). Karimishahri and Sharma (2016) analyzed molecular variation among populations of B. maydis in India, where two main genotypes were identified using RAPD markers, and reported as much as $94.8 \%$ polymorphic loci among single isolates from different locations. In our study, we found that values of NPL and PPL in the MAT1-2 population were significantly higher than those in the MAT1-1 population, suggesting that the MAT1-2 population is more diverse in terms of DNA polymorphism.

Our results also showed the value of $G_{S T}$ between MAT1-1 and MAT1-2 populations from different locations ranged from 0.0399 to 0.3044 , indicating the occurrence of genetic differentiation between the two mating type populations according to the classification of population differentiation for most regions (Nei 1978). The $G_{S T}$ value (Table 4) between MAT1-1 and MAT1-2 populations at FZ in Fujian Province was 0.3044 , suggesting approximately $70 \%$ of the genetic diversity originates from within populations. This finding is consistent with the high Shannon's information index (data not shown) but in contrast to that of the study by Li et al. (2016), in which low Shannon's information indices were calculated for other diverse pathogen populations. This may reflect that MAT1-1 and MAT1-2 populations in Fujian Province represent immigrant populations deriving from different geographic origins. Although ISSR markers indicated abundant polymorphic loci, the genetic diversity estimates reported in the present study should be confirmed using other molecular marker techniques and could further enhance our current understanding of the two mating type populations in Fujian Province.

Mating types of plant pathogens are also useful criteria for predicting the degree of virulence owing to sexual reproduction potential (Valent et al. 1991). Zhan et al. (2007) previously reported that a MAT1-1 population of the wheat fungal pathogen Mycosphaerella graminicola had a wider range of virulence on two wheat cultivars than a MAT1-2 population they tested. Genetic differentiation in neutral molecular marker loci between MAT1-1 and MAT1-2 populations was not obvious, consistent with the presupposition that diversity in virulence does not result from nonrandom genetic backgrounds or the presence of specific pathogenicity genes (Zhan et al. 2007). In our pathogenicity assays, MAT1-1 and MAT1-2 populations overcame all 11 sweet corn cultivars, with higher percentages of pathogenic isolates on both susceptible and resistant cultivars for both populations. Moreover, pathogenicity among $B$. maydis isolates from Fujian Province differed $(P<0.001)$ even though no difference in pathogenicity was detected between the two mating type populations $(P=0.625)$. These findings suggest that the pathogenicity of B. maydis in Fujian Province is not correlated with genetic diversity or mating types.

In the present study, high proportions of B. maydis isolates were pathogenic on all sweet corn cultivars tested, regardless of resistance or susceptibility, and despite the genetic differentiation among isolates observed using ISSR markers. This suggests that sexual recombination may not be necessary for pathogenicity and fitness in $B$. maydis. Local sweet corn cultivars are susceptible to infection by both mating types in Fujian Province in recent years (Dai et al. 2019). There are several reasons why many of the isolates we tested were pathogenic on sweet corn. In Fujian Province, most of the highyielding corn cultivars grown are sweet or waxy, and the majority are susceptible to SCLB (Dai et al. 2018b). Although many SCLBresistance recessive genes and quantitative trait loci have been identified in some corn inbred lines (Balint-Kurti et al. 2008; Kump et al. 2011; Zaitlin et al. 1993), these only provide partial resistance to SCLB. In addition, temperature and moisture conditions in Fujian Province favor infection of corn by B. maydis (Dai et al. 2016).
Continuous cropping of corn in this province may enhance the adaptability of the two mating type populations. Due to suitable weather conditions and crop profitability, corn (especially sweet and waxy corn) is planted more than twice in a season year in Fujian Province. Continuous cropping without a fallow period results in the build-up of a great adaptable $B$. maydis population. Further investigation is necessary to determine the role cropping practices play in the adaptability of the pathogen.

B. maydis MAT1-2 populations were more frequently detected compared with MAT1-1 populations in Fujian Province, although both populations were highly pathogenic on all tested local sweet corn cultivars. Because no sexual isolates were detected among the 198 isolates recovered from SCLB-symptomatic leaves collected from fields in this province, the SCLB fungus appears to be adaptive at overcoming the resistance of all tested sweet corn cultivars, even without sexual recombination. Nonetheless, the potential mechanism between sexual reproduction and pathogenicity should be further clarified, because this information will be important for devising integrated control measures for SCLB.

\section{Literature Cited}

Ahmadpour, A., Castell-Miller, C., Javan-Nikkhah, M., Naghavi, M. R., Dehkaei, F. P., Leng, Y., Puria, K. D., and Zhong, S. 2018. Population structure, genetic diversity, and sexual state of the rice brown spot pathogen Bipolaris oryzae from three Asian countries. Plant Pathol. 67:181-192.

Alcorn, J. L. 1975. Race-mating type associations in Australian populations of Cochliobolus heterostrophus. Plant Dis. Rep. 59:708-711.

Ali, H., Alam, S. S., Attanayake, R. N., Rahman, M., and Chen, W. 2012. Population structure and mating type distribution of the chickpea blight pathogen Ascochyta rabiei from Pakistan and the United States. J. Plant Pathol. 94:99-108.

Aylor, D. E., and Lukens, R. J. 1974. Liberation of Helminthosporium maydis spores by wind in the field. Phytopathology 64:1136-1138.

Balint-Kurti, P. J., Zwonitzer, J. C., Pè, M. E., Pea, G., Lee, M., and Cardinal, A. J. 2008. Identification of quantitative trait loci for resistance to southern leaf blight and days to anthesis in two maize recombinant inbred line populations. Phytopathology 98:315-320.

Bi, Y., Hu, J., Cui, X., Shao, J., Lu, X., Meng, Q., and Liu, X. 2014. Sexual reproduction increases the possibility that Phytophthora capsici will develop resistance to dimethomorph in China. Plant Pathol. 63:1365-1373.

Carson, M. L. 1998. Aggressiveness and perennation of isolates of Cochliobolus heterostrophus from North Carolina. Plant Dis. 82:1043-1047.

Cui, W., and Huang, W. 1985. Preliminary studies on the mating types of Cochliobolus heterostrophus in China. J. Hebei Agric. Univ. 8:10-16.

Dai, Y. L., Gan, L., Ruan, H. C., Shi, N. N., Du, Y. X., Chen, F. R., and Yang, X. J. 2018a. A PCR method to detect mating types of Cochliobolus heterostrophus. Can. J. Plant Pathol. 40:358-367.

Dai, Y. L., Gan, L., Ruan, H. C., Shi, N. N., Du, Y. X., Chen, F. R., and Yang, X. J. 2019. Genetic diversity and pathogenicity of different propiconazole-sensitive isolates of Bipolaris maydis in Fujian Province. Acta Phytopathol. Sin. 49: 64-74.

Dai, Y. L., Gan, L., Ruan, H. C., Shi, N. N., Du, Y. X., Liao, L., Wei, Z. X., Teng, Z. Y., Chen, F. R., and Yang, X. J. 2018b. Sensitivity of Cochliobolus heterostrophus to three demethylation inhibitor fungicides, propiconazole, diniconazole and prochloraz, and their efficacy against southern corn leaf blight in Fujian Province, China. Eur. J. Plant Pathol. 152:447-459.

Dai, Y. L., Yang, X. J., Gan, L., Chen, F. R., Ruan, H. C., Du, Y. X., Shi, N. N., and Gao, Z. M. 2016. First report of southern leaf blight caused by Cochliobolus heterostrophus on corn (Zea mays) in Fujian Province, China. Plant Dis. 100: 1781.

Fisher, D. E., and Hooker, A. L. 1975. Race, mating type, and virulence characteristics of the 1970 North American Helminthosporium maydis population. Plant Dis. Rep. 59:784-786.

Gafur, A., Tanaka, C., Ouchi, S., and Tsuda, M. 1997. A PCR-based method for mating type determination in Cochliobolus heterostrophus. Mycoscience 38: 455-458.

Gan, L., Dai, Y. L., Teng, Z. Y., Ruan, H. C., Du, Y. X., Shi, N. N., Chen, F. R., and Yang, X. J. 2016. Sensitivity of Bipolaris maydis to propiconazole, diniconazole and prochloraz in Fujian Province. Chin. J. Pestic. Sci. 18: 194-200.

Gao, J. X., Gao, Z. G., Zhang, X. F., Zhang, S., and Chen, L. 2010. A simple and feasible method for single-spore isolation of Exserohilum turcicum. Microbiol. China 37:1548-1550.

Glass, L. N., and Kuldau, G. A. 1992. Mating type and vegetative incompatibility in filamentous ascomycetes. Annu. Rev. Phytopathol. 30:201-224.

Gramaje, D., Armengol, J., and Ridgway, H. J. 2013. Genetic and virulence diversity, and mating type distribution of Togninia minima, causing grapevine trunk diseases in Spain. Eur. J. Plant Pathol. 135:727-743. 
Groenewald, M., Linde, C. C., Groenewald, J. Z., and Crous, P. W. 2008. Indirect evidence for sexual reproduction in Cercospora beticola populations from sugar beet. Plant Pathol. 57:25-32.

Grünwald, N. J., Goodwin, S. B., Milgroom, M. G., and Fry, W. E. 2003. Analysis of genotypic diversity data for populations of microorganisms. Phytopathology 93:738-746.

Gu, S. Q., Fan, Y. S., Li, P., and Dong, J. G. 2008. Optimization of ISSR reaction and genetic diversity analysis of Exserohilum turcicum. Acta Phytophylac. Sin. 35:427-432.

Kantety, R. V., Zeng, X., Bennetzen, J. L., and Zehr, B. E. 1995. Assessment of genetic diversity in dent and popcorn (Zea mays L.) inbred lines using intersimple sequence repeat (ISSR) amplification. Mol. Breed. 1:365-373.

Karimishahri, M. R., and Sharma, R. C. 2016. Genetic variation among isolates of Bipolaris maydis using RAPD-PCR. Ital. J. Mycol. 45:19-28.

Kim, H., Newell, A. D., Cota-Sieckmeyer, R. G., Rupe, J. C., Fakhoury, A. M., and Bluhm, B. H. 2013. Mating-type distribution and genetic diversity of Cercospora sojina populations on soybean from Arkansas: Evidence for potential sexual reproduction. Phytopathology 103:1045-1051.

Kong, L. X., Zhao, J. Y., Li, Q. S., Wang, L. S., and Luo, P. C. 2005. Identification and population dynamics of physiological races of Bipolaris maydis in Hebei. Acta Agric. Boreali-Sin. 20:90-93.

Kump, K. L., Bradbury, P. J., Wisser, R. J., Buckler, E. S., Belcher, A. R., Oropeza-Rosas, M. A., Zwonitzer, J. C., Kresovich, S., McMullen, M. D., Ware, D., Balint-Kurti, P. J., and Holland, J. B. 2011. Genome-wide association study of quantitative resistance to southern leaf blight in the maize nested association mapping population. Nat. Genet. 43:163-168.

Leonard, K. J. 1974. Bipolaris maydis race and mating type frequencies in North Carolina. Plant Dis. Rep. 58:529-531.

Lewontin, R. C. 1972. The apportionment of human diversity. J. Evol. Biol. 6: 381-398.

Li, J., Lu, L., Jia, Y., Wang, Q., Fukuta, Y., and Li, C. 2016. Characterization of field isolates of Magnaporthe oryzae with mating type, DNA fingerprinting, and pathogenicity assays. Plant Dis. 100:298-303.

Li, P., Cao, S., Dai, Y. L., Li, X. L., Xu, D. F., Guo, M., Pan, Y. M., and Guo, Z. M. 2012. Genetic diversity of Phytophthora capsici (Pythiaceae) isolates in Anhui Province of China based on ISSR-PCR markers. Genet. Mol. Res. 11: 4285-4296.

Linde, C. C., Zala, M., Ceccarelli, S., and McDonald, B. A. 2003. Further evidence for sexual reproduction in Rhynchosporium secalis based on distribution and frequency of mating-type alleles. Fungal Genet. Biol. 40:115-125.

May, G., Shaw, F., Badrane, H., and Vekemans, X. 1999. The signature of balancing selection: Fungal mating compatibility gene evolution. Proc. Natl. Acad. Sci. U.S.A. 96:9172-9177.

Mazáková, J., Zouhar, M., Ryšánek, P., Táborský, V., Hausvater, E., and Doležal, P. 2010. Mating type distribution of Phytophthora infestans (Mont.) de Bary in the Czech Republic in 2007 and 2008. Plant Prot. Sci. 46:89-97.

McDermott, J. M., and McDonald, B. A. 1993. Gene flow in plant pathosystems. Annu. Rev. Phytopathol. 31:353-373.

Milgroom, M. G. 1996. Recombination and the multilocus structure of fungal populations. Annu. Rev. Phytopathol. 34:457-477.

Nei, M. 1973. Analysis of gene diversity in subdivided populations. Proc. Natl. Acad. Sci. U.S.A. 70:3321-3323.

Nei, M. 1978. Estimation of average heterozygosity and genetic distance from a small number of individuals. Genetics 89:583-590.

Nelson, R. R., Ayers, J. E., Cole, H., Massie, L. B., and Forer, L. 1971. Distribution, race frequency, virulence, and mating type of isolates of Helminthosporium maydis in the northeastern united states in 1970. Plant Dis. Rep. 55:495-497.

Neuhauser, S., Huber, L., and Kirchmair, M. 2009. A DNA based method to detect the grapevine root-rotting fungus Roesleria subterranea in soil and root samples. Phytopathol. Mediterr. 48:553-559.

Ngoko, Z., Cardwell, K. F., Marasas, W. F. O., Wingfield, M. J., Ndemah, R., and Schulthess, F. 2002. Biological and physical constraints on maize production in the Humid Forest and Western Highlands of Cameroon. Eur. J. Plant Pathol. 108:893-902.
Nicholson, P., Rezanoor, H. N., and Su, H. 1993. Use of random amplified polymorphic DNA (RAPD) analysis and genetic fingerprinting to differentiate isolates of race $\mathrm{O}, \mathrm{C}$ and $\mathrm{T}$ of Bipolaris maydis. J. Phytopathol. 139:261-267.

Onaga, G., Wydra, K. D., Koopmann, B., Sere, Y., and Von, T. A. 2015 Population structure, pathogenicity and mating type distribution of Magnaporthe oryzae isolates from East Africa. Phytopathology 105: $1137-1145$

Reddy, J. M., Raoof, M. A., and Ulaganathan, K. 2012. Development of specific markers for identification of Indian isolates of Fusarium oxysporum f. sp. ricini. Eur. J. Plant Pathol. 134:713-719.

Salimath, S. S., Oliveira, A. C., Godwin, I. D., and Bennetzen, J. L. 1995. Assessment of genome origins and genetic diversity in the genus Eleusine with DNA markers. Genome 38:757-763.

Sharma, P., Samkumar, A., Rao, M., Singh, V. V., Prasad, L., Mishra, D. C., Bhattacharya, R., and Gupta, N. C. 2018. Genetic diversity studies based on morphological variability, pathogenicity and molecular phylogeny of the Sclerotinia sclerotiorum population from Indian mustard (Brassica juncea). Front. Microbiol. 9:1169.

Smith, D. R., Hooker, A. L., and Lim, S. M. 1970. Physiologic races of Helminthosporium maydis. Plant Dis. Rep. 54:819-822.

Tang, Q. Y. 2010. DPS ${ }^{\circledR}$ Data Processing System-Experimental Design, Statistical Analysis and Data Mining, 2nd Ed. Science Press, Beijing, China.

Tsukiboshi, T., Koga, H., and Uematsu, T. 1996. Races of Bipolaris maydis occurring in Japan and their pathogenicity to the rhm resistant corn line. Jpn. Agric. Res. Q. 30:91-96.

Turgeon, B. G., Bohlmann, H., Ciuffetti, L. M., Christiansen, S. K., Yang, G., Schäfer, W., and Yoder, O. C. 1993. Cloning and analysis of the mating type genes from Cochliobolus heterostrophus. Mol. Genet. Genomics 238: 270-284.

Tzeng, T. H., Lyngholm, L. K., Ford, C. F., and Bronson, C. R. 1992. A restriction fragment length polymorphism map and electrophoretic karyotype of the fungal maize pathogen Cochliobolus heterostrophus. Genetics 130:81-96.

Valent, B., Farrall, L., and Chumley, F. G. 1991. Magnaporthe grisea genes for pathogenicity and virulence identified through a series of backcrosses. Genetics 127:87-101.

Wang, L. Z., Kang, Z. Y., Wu, Y. X., Zhou, H. P., Mao, Z. C., and He, Y. Q. 2010 Preliminary identification of physiological races of Bipolaris maydis in Yunnan. J. Yunnan Univ. 32:352-357.

Wang, M., Wang, S., Ma, J., Yu, C., Gao, J., and Chen, J. 2017. Detection of Cochliobolus heterostrophus races in South China. J. Phytopathol. 165 681-691.

Wirsel, S., Horwitz, B., Yamaguchi, K., Yoder, O. C., and Turgeon, B. G. 1998 Single mating type-specific genes and their 3 '-UTRs control mating and fertility in Cochliobolus heterostrophus. Mol. Genet. Genomics 259:272-281.

Wirsel, S., Turgeon, B. G., and Yoder, O. C. 1996. Deletion of the Cochliobolus heterostrophus mating-type (MAT) locus promotes the function of MAT transgenes. Curr. Genet. 29:241-249.

Yeh, F. C., Yang, R. C., Boyle, T. B. J., Ye, Z. H., and Mao, J. X. 1999. POPGENE Version 1.32; The User-Friendly Shareware for Population Genetic Analysis.Molecular Biology and Biotechnology Centre, University of Alberta, Edmonton, AB, Canada.

Zaitlin, D., DeMars, S., and Ma, Y. 1993. Linkage of $r h m$, a recessive gene for resistance to southern corn leaf blight, to RFLP marker loci in maize (Zea mays) seedlings. Genome 36:555-564.

Zhan, J., Torriani, S. F. F., and McDonald, B. A. 2007. Significant difference in pathogenicity between MAT1-1 and MAT1-2 isolates in the wheat pathogen Mycosphaerella graminicola. Fungal Genet. Biol. 44:339-346.

Zhao, J. Y., Jiang, X. L., Jia, H. M., Li, S. C., Shi, J., and Zhang, H. J. 2012. Identification and evaluation of physiological races of Bipolaris maydis in Huanghuaihai region. J. Hebei Agric. Sci. 16:47-49.

Zwonitzer, J. C., Bubeck, D. M., Bhattramakki, D., Goodman, M. M., Arellano, C. and Balint-Kurti, P. J. 2009. Use of selection with recurrent backcrossing and QTL mapping to identify loci contributing to southern leaf blight resistance in a highly resistant maize line. Theor. Appl. Genet. 118:911-925. 